\title{
Fundamental Precision of Pulsar Timing
}

\author{
Roger S. Foster \\ Remote Sensing Division, Code 7213, Naval Research Laboratory, \\ Washington, DC 20375
}

\section{Background}

Although 13 years have passed since the first millisecond pulsar (MSP) was discovered (Backer et al. 1983), it still has the shortest known rotational period $(1.56 \mathrm{~ms})$. MSPs are nature's most stable clocks (Taylor 1991), with timing stabilities that rival atomic clocks on time scales beyond six months (Matsakis $\&$ Foster 1996). Specifically, the instantaneous measurement of the period of the original MSP is determined to a precision of $\sim 20$ attoseconds $\left(10^{-18} \mathrm{~s}\right)$. With such precision, we are able to predict the pulsar pulse arrival times to a small fraction of the rotational period years into the future. MSPs are powerful sources for use in fundamental astrometry and time keeping applications. Collectively, a population of MSPs distributed around the sky can be used to establish a nearly inertial space-time reference frame (e.g. Foster \& Backer 1990). Such a pulsar timing array (PTA) could be used to study drifts in Earth based atomic time scales, perturbation in the planetary ephemerides, relativistic corrections in the solar gravitational potential, and limit the energy density of a stochastic background of gravitational waves from the early universe.

In order to use pulsars to establish a fundamental space-time reference frame we need to assess the sources of errors and uncertainty in pulsar timing data and relate these errors back to the limits they place on making fundamental astrometric and metrology measurements. The techniques for making pulsar timing measurements are built on the efforts of many diverse programs, including the construction of large telescope observing facilities, the development of atomic clocks for high precision time keeping, and the implementation of a global system of satellites to allow time transfer. Several sources of uncertainty dominate pulsar timing measurements including intrinsic rotational noise associated with the pulsar, radiometer noise, individual pulse jitter, imbalance between orthogonal senses of polarization, propagation effects from the interstellar medium including both diffractive and refractive effects, and uncertainties due to time transfer via the Global Positioning System (GPS) of satellites.

A requirement for the successful implementation of a PTA is a good distribution of MSPs around the sky (Figure 1). The 1995 population has what appears to be a nearly "isotropic" distribution. The thirty identified field MSPs represent a rather local galactic population of stars that are typically at distances around $1 \mathrm{kpc}$ with a similar scale height. Further investigations of the distribution of these sources, after folding in the selection effects from the all sky surveys, are needed to properly infer the scale height of these old neutron stars. 
Field Millisecond Pulsars (1989)

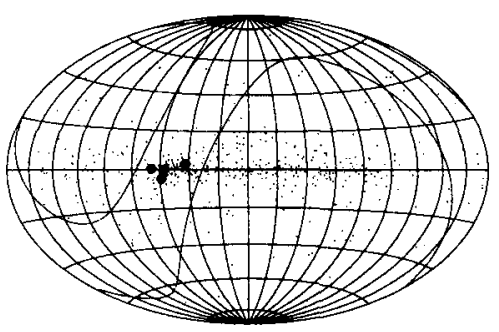

Field Millisecond Pulsars (1995)

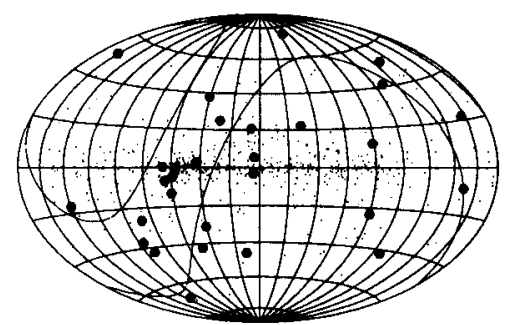

Figure 1. Figure 1 shows the comparative distributions of MSPs in the galactic field that were identified in 1989 with those that were known by the end of 1995 . The plots are in galactic coordinates and show the Arecibo declination limits at 0 and +38 degrees. Slow period pulsars are shown as small dots, while the MSPs are shown as large dots.

\section{Sources of Pulsar Timing Uncertainty}

The intrinsic rotational noise in neutron stars has recently been the focus of considerable interest, as it may represent the final limit in the long term stability of MSPs. The latest work focused on the two pulsars that have been timed the longest, PSR B1855+09 and PSR B1937+21(Kaspi et al. 1994). These two pulsars have been timed for at least 6 and 8 years respectively in timing programs at the Arecibo Observatory in Puerto Rico. Arzoumanian et al. (1994) examined the relationship between a pulsar's period and its period derivative for a large population of pulsars that includes both slow period and MSPs. They concluded that there is a good correlation between a pulsar's period derivative and its timing stability. The basic result is that pulsars with smaller period derivatives, and hence longer spin-down ages, generally are more stable clocks.

Another source of pulsar timing uncertainty has to do with the process of making measurements with large radio telescopes. Specifically, as the radiometer noise associated with the telescope and its detector hardware improves, the effects from intrinsic pulse jitter on the pulsar arrival time measurement become more significant. The critical observing,bandwidth that characterizes the cross-over point between the two regions can be parameterized by $\Delta B_{c}=\left(\frac{W}{\mu s}\right)^{2}\left(\frac{T_{s y s}}{K}\right)^{2}\left(\frac{\langle S\rangle}{m J_{y}}\right)^{-2}\left(\frac{G}{K / J_{y}}\right)^{-2}\left(\frac{P}{m s}\right)^{-3} \mathrm{kHz}$, where $W$ is the pulse width, $T_{s y s}$ is the system temperature of the receiver, $\langle S\rangle$ is the average flux density of the source, $G$ is the antenna gain, and $P$ is the pulse period. Pulse 
jitter can have powerful effects on the source of timing uncertainty for bright pulsars like PSR B1937+21. Using the $305 \mathrm{~m}$ antenna at Arecibo with only $350 \mathrm{kHz}$ of bandwidth and a feed centered at $1400 \mathrm{MHz}$ the amplitudes of the radiometer noise and the intrinsic pulse jitter are both about $100 \mathrm{~ns}$. Even on smaller telescopes, pulse jitter can be a limiting factor. For PSR B1937+21 using NRAO $43-\mathrm{m}$ antenna $\Delta B_{c}=50 \mathrm{MHz}$ at $1400 \mathrm{MHz}$.

Radio pulsars are typically strongly polarized steep-spectrum sources. Polarization imbalances in the antenna and mixing system can contribute significantly to the final measurement uncertainties if steps are not taken to properly account for gain variations in the two senses of orthogonal polarization. Some thought has been given to what procedures should be followed to optimally analyze pulse waveforms. Blaskiewicz (1991) has followed a principal components analysis (or Karhunen-Loeve Analysis) to select the optimal basis function for quantifying shape changes. Using these measurements, the fitted coefficients can be used to define error corrections. Further investigation is needed to see if it might be possible to apply a pulse Time of Arrival (TOA) error correction scheme to pulse arrivals when pulse jitter dominates the data and increased telescope observing time is not feasible.

To obtain the highest level of timing precision it is necessary to observe at radio frequencies above $1 \mathrm{GHz}$ to overcome propagation delays associated with interstellar turbulence. Backer (this volume) has observed frequency dependent changes in DM due to the different effective volumes of interstellar space sampled by radio telescope beams at different radio frequencies. This result points out that the effective DM is frequency dependent and cannot be corrected by a simple $\nu^{2}$ scaling. Observing at the highest practical frequency, as long as the pulsar flux density is above a minimal detection threshold, requires that the maximum bandwidth and lowest system temperatures be obtained with the radio telescope. Multiple frequency observations are useful to monitor for dispersion measure variations to apply first order corrections.

\section{An Inertial Space-time Reference Frame}

Pulsars offer a unique set of galactic sources for establishing an inertial spacetime reference frame. Using observations of MSPs (of which more than 75 percent are in binary systems with white dwarf companions) in three distinct reference frames, all three frames can be linked together using the same fundamental sources. Three techniques for observing MSPs are required (one for each reference frame): (1) radio pulsar timing to measure the pulsar position in the dynamical reference frame, (2) Very Long Baseline Interferometric (VLBI) observations of the radio pulsars to measure the radio source position, and (3) optical observations of the white dwarf companions to tie the optical reference frame with the other two frames.

The precision to which these three reference frames can be tied varies depending on the systems used. For example, a position has been obtained from pulsar timing to an angular precision of 0.06 mas after 8.2 years of observations on PSR B1937+21 (Kaspi et al. 1994, and Table 1). Long-term observations of the radio reference frame have established radio source positions to a precision of 0.05 mas (Johnston et al. 1995). The current planetary ephemeris, the DE200, 
has a rotation between the radio (IERS) and dynamical reference frame of 13 mas (Folker et al. 1993). An improved ephemeris, the DE403, has a rotation of about 3 mas between the two frames (Standish 1995). The rotation between the radio and optical frames is much worse. The current uncertainty between the FK5 and IERS frames is about 200 mas. The use of Hipparcos astrometric data should greatly improve the uncertainty between these two frames providing an error $\epsilon=0.5+0.5 *\left(t_{\text {years }}-1991.25\right)$ mas, where $t$ is the date of the second frame observations. In addition, Hubble and/or ground-based astrometric observations of the white dwarf companions to binary MSPs may be able to have positions measured to an accuracy of 30 mas. Unlike other astrometric techniques for determining position, such as planetary ranging observations, pulsar timing techniques are not confined to the ecliptic.

Table 1. Pulsar Precision

\begin{tabular}{lrrrrl}
\hline \hline $\begin{array}{l}\text { Pulsar } \\
\text { Name }\end{array}$ & $\begin{array}{r}\text { Observ. } \\
\text { Duration } \\
\text { (years) }\end{array}$ & $\begin{array}{r}\text { Timing } \\
\text { Prec. } \\
\text { (us) }\end{array}$ & $\begin{array}{r}\text { RA } \\
\text { (mas) }\end{array}$ & $\begin{array}{r}\text { DEC } \\
\text { Prec. } \\
\text { (mas) }\end{array}$ & $\overline{\text { Reference }}$ \\
\hline B1937+21 & 8.2 & 0.2 & 0.03 & 0.06 & Kaspi et al. 1994 \\
B1855+09 & 6.9 & 1.0 & 0.07 & 0.12 & Kaspi et al. 1994 \\
J1713+0747 & 1.8 & 0.4 & 0.2 & 0.3 & Camilo et al. 1994 \\
\hline
\end{tabular}

\section{Conclusions}

With the dramatic improvements in terrestrial clocks since 1990 and the discovery of numerous millisecond pulsars in the galactic field, pulsar timing provides a powerful tool for a variety of applications. The creation of a pulsar space-time reference frame is a goal that will require coordinated work from a number of different observers and observatories.

\section{References}

Arzoumanian, Z., et al. 1994, ApJ, 422, 671

Backer, D. C., et al. 1982, Nature, 300, 615

Blaskiewicz, M. 1991, Ph.D. Thesis Cornell University

Camilo, F., Foster, R. S., Wolszczan, A. 1994, ApJ, 437, L39

Folker, W. M., et al. 1994, å, 287, 279

Foster, R. S. \& Backer, D. C. 1990, ApJ, 361, 300

Johnston, K. J., et al. 1995, AJ, 110, 880

Kaspi, V. M., Taylor, J. H., Ryba, M. F. 1994, ApJ, 428, 713

Matsakis, D. M. \& Foster, R. S. 1996, Amazing Light, a Festschrift in Honor of Charles H. Townes, Springer-Verlag Press

Standish, E. M., Jr. 1995, JPL IOM 314, 10-124

Taylor, J. H. 1991, IEEE, Proceedings, 79, 1054 